\section{A Constructive Approach to Local Stabilization of Nonlinear Systems by Dynamic Output Feedback}

\author{
Pengnian Chen, Daizhan Cheng, and Zhong-Ping Jiang
}

\begin{abstract}
The note considers the problem of local stabilization of nonlinear systems by dynamic output feedback. A new concept, namely, local uniform observability of feedback control law, is introduced. The main result is that if a nonlinear system is $N$ th-order approximately stabilizable by a locally uniformly observable state feedback, then it is stabilizable by dynamic output feedback. Based on the approximate stability, a constructive method for designing dynamic compensators is presented. The design of the dynamic compensators is beyond the separation principle and can handle systems whose linearization might be uncontrollable and/or unobservable. An example of nonminimum phase nonlinear systems is presented to illustrate the utility of the results.
\end{abstract}

Index Terms-Dynamic output feedback, local uniform observability, nonlinear systems, nonminimum phase systems, stabilization.

\section{INTRODUCTION}

In this note, we study the problem of dynamic output feedback stabilization of a nonlinear system of the form

$$
\begin{aligned}
& \dot{x}=f(x)+g(x) u \\
& y=h(x)
\end{aligned}
$$

where $x \in U \subset R^{n}, u, y \in R^{m} ; f \in C^{\infty}\left(U, R^{n}\right), f(0)=0$, $g \in C^{\infty}\left(U, R^{n \times m}\right), h \in C^{\infty}\left(U, R^{m}\right), h(0)=0$, and $U$ is a neighborhood of $x=0$.

Stabilization by dynamic output feedback is a fundamentally important issue in the theory of nonlinear systems and has been extensively studied in the past two decades. According to the region of attraction, it can be classified into three categories: local, semi-global and global stabilization problems. Indeed, the semi-global and global stabilization can guarantee that closed loop systems have sufficiently large regions of attraction and are of great interest (see, for instance, [7], [10], [11], and [13]). However, the local stabilization of nonlinear systems is fundamental and important ( see, for instance, [1], [2], and [15] ). In this note, we only consider the problem of local stabilization.

The nonlinear systems that have been dealt with so far in the study of local dynamic output feedback stabilization are mainly of two classes: One is completely uniformly observable systems (see, for instance, [7] and [15]); the other is minimum phase nonlinear systems. that a nonlinear system observable, then it is stabilizable feedback stabilization of uniformly observable. In [3], the authors applied the center manifold theory to study the problem of dynamic output feedback stabilization. In particular, [3] stabilization by using presents an example that is not stabilizable by static output feedback, but is stabilizable by dynamic output feedback. It is easy to see that the example in [3] is a minimum

Manuscript received September 13, 2004; revised September 20, 2005 and November 16, 2005. Recommended by Associate Editor D. Nesic. This work was supported in part by the National Natural Science Foundation of China under grant 60274008,60274010 , and in part by U.S. NSF grants ECS-0093176, OISE-0408925 and DMS-0504462.

P. Chen is with the Department of Mathematics, China Institute of Metrology, Hangzhou 310018, P. R. China (e-mail: pnchen@mail.hz.zj.cn).

D. Cheng is with the Institute of Systems Science, Chinese Academy of Sciences, Beijing 100080, P. R. China (e-mail: dcheng@mail.iss.ac.cn).

Z.-P. Jiang is with the Department of Electrical and Computer Engineering, Polytechnic University, Brooklyn, NY 11201 USA (e-mail: zjiang@ control. poly.edu).

Digital Object Identifier 10.1109/TAC.2006.878753 phase nonlinear system with relative degree one, whose zero dynamics is fifth-order approximately stable (see, for instance, [5], [6], and [8]. Recent papers [4] and [5], motivated by the example, have proved that a minimum phase nonlinear system is stabilizable by dynamic output feedback if its zero dynamics is $N$ th-order approximately stable for some positive integer $N$. The nonlinear systems studied in this note are more general than those in previous study, which may be of nonminimum phase and may have unstabilizable and/or undetectable linear approximations. References [16] and [12] have already considered nonlinear systems with unstabilizable and/or undetectable linear approximation, but those nonlinear systems have special forms.

Two key concepts used in this note are approximate stability and local uniform observability of a state feedback law. The concept of the approximate stability is well-known in the stability theory of differential equations (see, for instance, [8]), and, recently, has been applied to stabilization of nonlinear systems (see, for instance, [4]-[6]). The concept of local uniform observability of a feedback law (or a function) is introduced in this note, which is a local version of the concept of uniform complete observability of a function introduced in [14]. In this note, based on these two concepts, we present a constructive approach to local dynamic output feedback stabilization. The approach can handle a wide class of nonlinear systems which can not be handled by existing methods. Roughly speaking, the main result claims that if there exists a state feedback law such that the closed-loop system is $N$ th-order approximately stable and the state feedback law is locally uniformly observable, then the system is stabilizable by dynamic output feedback.

The note is organized as follows. Section II introduces the concepts of the approximate stability and the local uniform observability. Section III is the main result of this note. A constructive technique for designing dynamic compensators is presented. In Section IV, an example of nonminimum phase and not locally uniformly observable nonlinear system is discussed. Section V contains some concluding remarks.

\section{APPROXIMATE STABILITY AND LOCAL UNIFORM OBSERVABILITY}

First we review the concept of approximate stability (see, for instance, [5], [6], and [8]). Consider the differential equation

$$
\dot{x}=F(x) \quad F(0)=0, \quad x \in U
$$

where $U \subset R^{n}$ is an open neighborhood of $x=0$, and $F \in C^{\infty}\left(U, R^{n}\right)$.

Throughout the note, stability of a system always refers to the stability of the zero solution of the system.

Definition 2.1: System (2) is said to be $N$ th-order approximately stable, if for any $C^{1}$ function $\phi(x)$ satisfying $\phi(x)=O\left(\|x\|^{N+1}\right)$, the differential equation

$$
\dot{x}=F(x)+\phi(x)
$$

is locally asymptotically stable.

For convenience, we introduce the following Malkin's stability theorem without proof, whose proof can be found in [5] and [8]. Consider the nonlinear system

$$
\begin{aligned}
\dot{x} & =F(x, w) \\
\dot{w} & =A w+H(x, w)
\end{aligned}
$$

where $x \in R^{n}, w \in R^{m}$, and $A \in R^{m \times m} ; F(x, w)$ and $H(x, w)$ defined on a neighborhood of $(x, w)=(0,0)$, are $C^{2}$ mappings with $F(0,0)=0$ and $H(0,0)=0$. 
Theorem 2.1: (Malkin's stability theorem) System (4) is locally asymptotically stable if the following conditions hold.

i) $\operatorname{Re} \sigma(A)<0$.

ii) the zero solution of the differential equation

$$
\dot{x}=F(x, 0)
$$

is $N$ th-order approximately stable for some positive integer $N$. iii) $H(x, 0)=O\left(\|x\|^{N+1}\right)$, and $H(x, w)=O\left(\|(x, w)\|^{2}\right)$.

We now introduce the concept of local uniform complete observability of a function, which is a local revision of the concept of uniform complete observability introduced in [14]. For (1), we define

$$
\begin{aligned}
y_{0} \triangleq & h(x) \\
y_{1} & =y_{1}\left(x, u_{0}\right) \\
\triangleq & \left(\frac{\partial}{\partial x} y_{0}\right)\left(f(x)+g(x) u_{0}\right) \\
y_{i+1}= & y_{i+1}\left(x, u_{0}, u_{1}, \ldots, u_{i}\right) \\
\triangleq & {\left[\frac{\partial}{\partial x} y_{i}\left(x, u_{0}, u_{1}, \ldots, u_{i-1}\right)\right]\left(f(x)+g(x) u_{0}\right) } \\
& +\sum_{j=0}^{i-1}\left[\frac{\partial}{\partial u_{j}} y_{i}\left(x, u_{0}, u_{1}, \ldots, u_{i-1}\right)\right] u_{j+1}, \\
& i=1,2, \ldots
\end{aligned}
$$

where $u_{j} \in R^{m}, j=0,1, \ldots$.

Definition 2.2: Let a function $\alpha(x)$ with $\alpha(0)=0$ be defined on some neighborhood of $x=0 . \alpha(x)$ is said to be locally uniformly observable with respect to (1) if there exist two non-negative integers $k_{1}$ and $k_{2}$ and a $C^{\infty}$ function $\phi$, vanishing at the origin, such that

$$
\alpha(x)=\phi\left(y_{0}, y_{1}, \ldots, y_{k_{1}}, u_{0}, u_{1}, \ldots, u_{k_{2}}\right)
$$

holds on a neighborhood of $x=0, u_{0}=0, u_{1}=0, \ldots, u_{k_{2}}=0$.

\section{Dynamic OUtPut FEedBaCK StabiLization}

In order to simplify notations and expressions in the sequel, we assume $m=1$ in (1) and system (1) has relative degree $r$ at the origin. Based on the theory of input-output linearization [9], (1) can be locally transformed into

$$
\begin{aligned}
\dot{z} & =f_{0}(z, \xi)+g_{0}(z, \xi) u \\
\dot{\xi} & =A_{0} \xi+B_{0}\left(F_{0}(z, \xi)+G(z, \xi) u\right) \\
y & =C_{0} \xi
\end{aligned}
$$

where

$$
\begin{aligned}
A_{0} & =\left(\begin{array}{ccccc}
0 & 1 & 0 & \cdots & 0 \\
& & \cdots & & \\
0 & 0 & 0 & \cdots & 1 \\
0 & 0 & 0 & \cdots & 0
\end{array}\right) \in R^{r \times r} \\
B_{0} & =\left(\begin{array}{c}
0 \\
\cdots \\
0 \\
1
\end{array}\right) \in R^{r \times 1} \quad C_{0}=(1,0, \ldots, 0) \in R^{1 \times r}
\end{aligned}
$$

where $z \in R^{n-r}, \xi=\left(\xi_{1}, \xi_{2}, \ldots, \xi_{r}\right)^{T} \in R^{r} ; f_{0}, g_{0}, F_{0}$ and $G$ are $C^{\infty}$ functions defined on a neighborhood of $z=0$ and $\xi=0$, with $f_{0}$ and $F_{0}$ vanishing at $z=0, \xi=0$ and $G(0,0) \neq 0$.

In this section, we assume without loss of generality that $x=\left(z^{T}, \xi^{T}\right)^{T}, f(x)=\left(\left(f_{0}(z, \xi)\right)^{T},\left(A_{0} \xi+B_{0} F_{0}(z, \xi)^{T}\right)^{T}\right.$, and $g(x)=\left(\left(g_{0}(z, \xi)\right)^{T},\left(B_{0} G(z, \xi)\right)^{T}\right)^{T}$.
Theorem 3.1: If there exists a $C^{\infty}$ function $\alpha(x)$ with $\alpha(0)=0$, defined on a neighborhood of $x=0$, such that the following conditions hold.

i) The system

$$
\dot{x}=f(x)+g(x) \alpha(x)
$$

is $N$ th-order approximately stable.

ii) $\alpha(x)$ is locally uniformly observable with respect to (1), i.e., there exists a $C^{\infty}$ function $\phi$, vanishing at the origin, such that

$$
\alpha(x)=\phi\left(y_{0}, y_{1}, \ldots, y_{k_{1}}, u_{0}, u_{1}, \ldots, u_{k_{2}}\right) .
$$

Then system (1) is locally asymptotically stabilizable by dynamic output feedback.

Proof: It suffices to show that system (8) is stabilizable by dynamic output feedback. The proof is composed of the following five steps to construct a desired dynamic compensator for (8).

Step 1. Introduction of an auxiliary dynamic system.

The dynamic system is

$$
\begin{aligned}
\dot{\lambda}_{1} & =\lambda_{2} \\
\cdots & \\
\dot{\lambda}_{l-1} & =\lambda_{l} \\
\dot{\lambda}_{l} & =\tilde{u} \\
u & =\lambda_{1}
\end{aligned}
$$

where $\lambda_{i} \in R, i=1,2, \ldots, l ; u=\lambda_{1}$ is the output, $\tilde{u}$ the input; $l$ is a positive integer to be determined later.

Step 2. Two transformations for the composite system of (8) and (12).

In this step, we perform two transformations for the composite system of (8) and (12). One transformation is used to construct a stabilizing controller of the composite system (8) and (12); the other transformation is used to construct an observer for $y^{(1)}, y^{(2)}, \ldots, y^{\left(k_{1}\right)}$. In order to determine $l$ in (12), we need to perform the two transformations simultaneously.

Let

$$
\begin{aligned}
\eta_{1} & =\lambda_{1}-\alpha(z, \xi) \\
\zeta_{1} & =F_{0}(z, \xi)+G(z, \xi) \lambda_{1}
\end{aligned}
$$

where $\alpha(z, \xi)=\alpha(x)$. Then, we have

$$
\begin{aligned}
& \dot{\eta}_{1}=F_{1}\left(z, \xi, \eta_{1}\right)+\lambda_{2} \\
& \dot{\zeta}_{1}=\bar{F}_{1}\left(z, \xi, \zeta_{1}\right)+G(z, \xi) \lambda_{2}
\end{aligned}
$$

where

$$
\begin{aligned}
& F_{1}\left(z, \xi, \eta_{1}\right) \\
= & -\left\{\frac{\partial}{\partial z} \alpha(z, \xi)\left[f_{0}(z, \xi)+g_{0}(z, \xi) \lambda_{1}\right]+\frac{\partial}{\partial \xi} \alpha(z, \xi)\right. \\
& {\left.\left[A_{0} \xi+B_{0}\left(F_{0}(z, \xi)+G(z, \xi) \lambda_{1}\right)\right]\right\}\left.\right|_{\lambda_{1}=\eta_{1}+\alpha(z, \xi)} }
\end{aligned}
$$

and $\bar{F}_{1}$ is defined similarly. Inductively, we can define

$$
\begin{aligned}
\eta_{i} & =F_{i-1}\left(z, \xi, \eta_{1}, \ldots, \eta_{i-1}\right)+\lambda_{i} \\
\zeta_{i} & =\bar{F}_{i-1}\left(z, \xi, \zeta_{1}, \ldots, \zeta_{i-1}\right)+G(z, \xi) \lambda_{i}, \quad i=2,3, \ldots, l
\end{aligned}
$$


and obtain

$$
\begin{aligned}
\dot{\eta}_{i} & =F_{i}\left(z, \xi, \eta_{1}, \ldots, \eta_{i}\right)+\lambda_{i+1}, \\
\dot{\zeta}_{i} & =\bar{F}_{i}\left(z, \xi, \zeta_{1}, \ldots, \zeta_{i}\right)+G(z, \xi) \lambda_{i+1}, \quad i=2,3, \ldots, l
\end{aligned}
$$

where $\lambda_{l+1}=\tilde{u}$.

Now, we express $F_{i}\left(z, \xi, \eta_{1}, \ldots, \eta_{i}\right)$ as a sum of three parts

$$
F_{i}\left(z, \xi, \eta_{1}, \ldots, \eta_{i}\right)=\sum_{j=1}^{i} a_{i j} \eta_{j}+p_{i}(z, \xi)+\varphi_{i}\left(z, \xi, \eta_{1}, \ldots, \eta_{i}\right)
$$

where $a_{i j}, j=1,2, \ldots, i$ are constants; $p_{i}(z, \xi)$ is a polynomial of degree less than or equal to $N$; and $\varphi_{i}\left(z, \xi, \eta_{1}, \ldots, \eta_{i}\right)$ has the properties that

$$
\begin{aligned}
\varphi_{i}\left(z, \xi, \eta_{1}, \ldots, \eta_{i}\right) & =O\left(\left\|\left(z, \xi, \eta_{1}, \ldots, \eta_{i}\right)\right\|^{2}\right) \\
\varphi_{i}(z, \xi, 0, \ldots, 0) & =O\left(\|(z, \xi)\|^{N+1}\right) .
\end{aligned}
$$

Due to (13) and (16), $\zeta_{i}$ is a function of $z, \xi, \eta_{1}, \ldots, \eta_{i}$. Hence, we can also express $\bar{F}_{i}\left(z, \xi, \zeta_{1}, \ldots, \zeta_{i}\right)$ as a sum of three parts

$$
\begin{aligned}
\bar{F}_{i}\left(z, \xi, \zeta_{1}, \ldots, \zeta_{i}\right) & \\
= & \sum_{j=1}^{i} \bar{a}_{i j} \zeta_{j}+\bar{p}_{i}(z, \xi)+\bar{\varphi}_{i}\left(z, \xi, \eta_{1}, \ldots, \eta_{i}\right)
\end{aligned}
$$

where $\bar{a}_{i j}, j=1,2, \ldots, i$, are constants; $\bar{p}_{i}(z, \xi)$ is a polynomial of degree less than or equal to $N ; \bar{\varphi}_{i}\left(z, \xi, \eta_{1}, \ldots, \eta_{i}\right)$ has the properties that

$$
\begin{aligned}
\bar{\varphi}_{i}\left(z, \xi, \eta_{1}, \ldots, \eta_{i}\right) & =O\left(\left\|\left(z, \xi, \eta_{1}, \ldots, \eta_{i}\right)\right\|^{2}\right) \\
\bar{\varphi}_{i}(z, \xi, 0, \ldots, 0) & =O\left(\|(z, \xi)\|^{N+1}\right) .
\end{aligned}
$$

Note that independent variables of $\bar{\varphi}_{i}$ in (20) are $z, \xi, \eta_{1}, \ldots, \eta_{i}$, which are the same as those of $\varphi_{i}$ in (18).

Let $l^{*}=\max \left\{k_{1}-r+1, k_{2}+1\right\}$, where $k_{1}$ and $k_{2}$ are defined in (11). Since $p_{i}(z, \xi)$ and $\bar{p}_{i}(z, \xi), i=1,2, \ldots$, are all polynomials of degree less than or equal to $N$, by linear algebra, there exists a positive integer $l \geq l^{*}$ and a set of constants $c_{i}, i=1,2, \ldots, l-1$, such that

$$
p_{l}(z, \xi)=\sum_{i=1}^{l-1} c_{i} p_{i}(z, \xi) \quad \bar{p}_{l}(z, \xi)=\sum_{i=1}^{l-1} c_{i} \bar{p}_{i}(z, \xi) .
$$

Take $l \geq l^{*}$ as the smallest positive integer that makes (22) hold. Then, from (16), (18), and (20), we obtain (23), as shown at the bottom of the page.

Let

$$
\tilde{u}=\sum_{i=1}^{l-1} c_{i} \lambda_{i+1}+v
$$

where $v$ is a new input. Then by (17), (18), (20), (23), and (24), we have

$$
\begin{aligned}
\dot{\eta}_{l} & =F_{l}\left(z, \xi, \eta_{1}, \ldots, \eta_{l}\right)+\tilde{u} \\
& =\sum_{i=1}^{l} a_{i} \eta_{i}+\varphi\left(z, \xi, \eta_{1}, \ldots, \eta_{l}\right)+v \\
\dot{\zeta}_{l} & =\bar{F}_{l}\left(z, \xi, \theta_{1}, \ldots, \theta_{l}\right)+G(z, \xi) \tilde{u} \\
& =\sum_{i=1}^{l} \bar{a}_{i} \zeta_{i}+\bar{\varphi}\left(z, \xi, \eta_{1}, \ldots, \eta_{l}\right)+G(z, \xi) v
\end{aligned}
$$

where $a_{i}$ and $\bar{a}_{i}, i=1,2, \ldots, l$, are some constants, and

$$
\begin{aligned}
& \varphi\left(z, \xi, \eta_{1}, \ldots, \eta_{l}\right) \\
& \quad=\varphi_{l}\left(z, \xi, \eta_{1}, \ldots, \eta_{l}\right)-\sum_{i=1}^{l-1} c_{i} \varphi_{i}\left(z, \xi, \eta_{1}, \ldots, \eta_{i}\right) \\
& \bar{\varphi}\left(z, \xi, \eta_{1}, \ldots, \eta_{l}\right) \\
& \quad=\bar{\varphi}_{l}\left(z, \xi, \eta_{1}, \ldots, \eta_{l}\right)-\sum_{i=1}^{l-1} c_{i} \bar{\varphi}_{i}\left(z, \xi, \eta_{1}, \ldots, \eta_{i}\right) .
\end{aligned}
$$

It follows from (19), (21), and (26) that

$$
\begin{aligned}
\varphi\left(z, \xi, \eta_{1}, \ldots, \eta_{l}\right) & =O\left(\left\|\left(z, \xi, \eta_{1}, \ldots, \eta_{i}\right)\right\|^{2}\right) \\
\varphi(z, \xi, 0, \ldots, 0) & =O\left(\|(z, \xi)\|^{N+1}\right)
\end{aligned}
$$

and

$$
\begin{aligned}
\bar{\varphi}\left(z, \xi, \eta_{1}, \ldots, \eta_{l}\right) & =O\left(\left\|\left(z, \xi, \eta_{1}, \ldots, \eta_{i}\right)\right\|^{2}\right) \\
\bar{\varphi}(z, \xi, 0, \ldots, 0) & =O\left(\|(z, \xi)\|^{N+1}\right) .
\end{aligned}
$$

Let $\eta=\left(\eta_{1}, \eta_{2}, \ldots, \eta_{l}\right)^{T}$ and $\bar{\xi}=\left(\bar{\xi}_{1}, \bar{\xi}_{2}, \ldots, \bar{\xi}_{r+l}\right)^{T}$ with $\bar{\xi}_{i}=$ $\xi_{i}, i=1,2, \ldots, r$, and $\bar{\xi}_{r+j}=\zeta_{j}, j=1,2, \ldots, l$. Then, by (13), (14), (16), (17), and (25), the composite system (8) and (12) can be transformed into two forms

$$
\begin{aligned}
\dot{z} & =f_{0}(z, \xi)+g_{0}(z, \xi)\left(\alpha(z, \xi)+\eta_{1}\right) \\
\dot{\xi} & =A_{0} \xi+B_{0}\left(F_{0}(z, x)+G(z, x)\left(\alpha(z, \xi)+\eta_{1}\right)\right) \\
\dot{\eta} & =A_{1} \eta+B_{1}\left(\varphi\left(z, \xi, \eta_{1}, \ldots, \eta_{l}\right)+v\right) \\
y & =C_{0} \xi
\end{aligned}
$$

where $\left(A_{1}, B_{1}\right)$ is in the controllable canonical form and

$$
\begin{aligned}
\dot{z} & =f_{0}(z, \xi)+g_{0}(z, \xi) G^{-1}(z, \xi)\left(\zeta_{1}-F_{0}(z, \xi)\right) \\
\dot{\bar{\xi}} & =\bar{A}_{1} \bar{\xi}+\bar{B}_{1}\left(\bar{\varphi}\left(z, \xi, \eta_{1}, \ldots, \eta_{l}\right)+G(z, \xi) v\right) \\
y & =\bar{C}_{1} \bar{\xi}
\end{aligned}
$$

where $\left(\bar{C}_{1}, \bar{A}_{1}\right)$ is in the observable canonical form.

Step 3. Construction of a stabilizing controller for system (29).

Since $\left(A_{1}, B_{1}\right)$ is in the controllable canonical form, the system

$$
\dot{\eta}=A_{1} \eta+B_{1} v
$$

$$
\begin{aligned}
& p_{l}(z, \xi)=\sum_{i=1}^{l-1} c_{i}\left(\eta_{i+1}-\sum_{j=1}^{i} a_{i j} \eta_{j}-\varphi_{i}\left(z, \xi, \eta_{1}, \ldots, \eta_{i}\right)-\lambda_{i+1}\right) \\
& \bar{p}_{l}(z, \xi)=\sum_{i=1}^{l-1} c_{i}\left(\zeta_{i+1}-\sum_{j=1}^{i} \bar{a}_{i j} \zeta_{j}-\bar{\varphi}_{i}\left(z, \xi, \eta_{1}, \ldots, \eta_{i}\right)-G(z, \xi) \lambda_{i+1}\right)
\end{aligned}
$$


is controllable and observable if we take $\eta_{1}$ as an output. Therefore, there exists a linear dynamic compensator

$$
\begin{aligned}
& \dot{\psi}=W \psi+L \eta_{1} \\
& v=Q \psi
\end{aligned}
$$

where $W, L$, and $Q$ are matrices with suitable dimensions, such that the closed-loop system

$$
\left(\begin{array}{c}
\dot{\eta} \\
\dot{\psi}
\end{array}\right)=\left(\begin{array}{cc}
A_{1} & B_{1} Q \\
L C_{1} & W
\end{array}\right)\left(\begin{array}{l}
\eta \\
\psi
\end{array}\right)
$$

is asymptotically stable, where $C_{1}=\left(\begin{array}{llll}1 & 0 & \cdots & 0\end{array}\right) \in R^{1 \times l}$. Let $w_{1}=\left(\eta^{T}, \psi^{T}\right)^{T}$,

$$
\begin{aligned}
F\left(x, \eta_{1}\right)= & \left(\begin{array}{c}
f_{0}(z, \xi) \\
A_{0} \xi+B_{0} F_{0}(z, \xi)
\end{array}\right) \\
& +\left(\begin{array}{c}
g_{0}(z, \xi)\left(\alpha(z, \xi)+\eta_{1}\right) \\
B_{0} G(z, x)\left(\alpha(z, \xi)+\eta_{1}\right)
\end{array}\right) \\
H_{1}\left(x, w_{1}\right)= & \left(\begin{array}{c}
B_{1} \varphi\left(z, \xi, \eta_{1}, \ldots, \eta_{l}\right) \\
0
\end{array}\right)
\end{aligned}
$$

and

$$
\bar{A}=\left(\begin{array}{cc}
A_{1} & B_{1} Q \\
L C_{1} & W
\end{array}\right)
$$

Then the composite system of (29) and (32) can be written as

$$
\begin{aligned}
\dot{x} & =F\left(x, \eta_{1}\right) \\
\dot{w}_{1} & =\bar{A} w_{1}+H_{1}\left(x, w_{1}\right) .
\end{aligned}
$$

Since $F(x, 0)=f(x)+g(x) \alpha(x)$, by condition i) of Theorem 3.1, the system

$$
\dot{x}=F(x, 0)
$$

is $N$ th-order approximately stable. On the other hand, we can see that

$$
H_{1}\left(x, w_{1}\right)=O\left(\|\left(x, w_{1}\right)\right) \|^{2} \quad H_{1}(x, 0)=O\left(\|x\|^{N+1}\right) .
$$

Then, by Theorem 2.1, with $w=w_{1}, A=\bar{A}$, and $H(x, w)=$ $H_{1}\left(x, w_{1}\right)$, system (37) is locally asymptotically stable. This means that (32) is also a stabilizing controller for (29). However, the controller (32) is difficult to be realized, since it contains $\eta_{1}=\lambda_{1}-\alpha(x)$, which cannot be measured directly.

Step 4. Construction of an observer for $y^{(1)}, y^{(2)}, \ldots, y^{\left(k_{1}\right)}$

Since $\left(\bar{C}_{1}, \bar{A}_{1}\right)$ is observable, there exists an $(r+l) \times 1$ matrix $\bar{L}$ such that

$$
\bar{A}_{1}-\bar{L} \bar{C}_{1}
$$

is a Hurwitz matrix. Let $\theta=\left(\theta_{1}, \theta_{2}, \ldots, \theta_{r+l}\right)^{T}$. We construct an observer for $y^{(1)}, y^{(2)}, \ldots, y^{\left(k_{1}\right)}$ as follows

$$
\dot{\theta}=\bar{A}_{1} \theta+\bar{L}\left(y-\bar{C}_{1} \theta\right)+\bar{B}_{1} G(0,0) Q \psi \text {. }
$$

Step 5. Construction of the whole dynamic compensator for (8).
Since $u_{j}, j=0,1, \ldots$, in (4), are independent variables, we may let

$$
u_{k}=\lambda_{k+1}, \quad k=0,1, \ldots, k_{2} .
$$

Then, from the second equality of (13) and the second equality of (16), we have

$$
y_{k}=\bar{\xi}_{k+1}, \quad k=0,1, \ldots, k_{1}
$$

Since $l \geq l^{*}=\max \left\{k_{1}-r+1, k_{2}+1\right\},(41)$ and (42) are well defined. Let $\lambda=\left(\lambda_{1}, \lambda_{2}, \ldots, \lambda_{l}\right)^{T}$. Let $\tilde{\phi}(\bar{\xi}, \lambda)$ be the function obtained from $\phi\left(y_{0}, y_{1}, \ldots, y_{k_{1}}, u_{0}, u_{1}, \ldots, u_{k_{2}}\right)$ in which $y_{k}$ is replaced by $\bar{\xi}_{k+1}, k=0,1, \ldots, k_{1}$, and $u_{k}$ is replaced by $\lambda_{k+1}, k=0,1, \ldots, k_{2}$. $\tilde{\phi}(\bar{\xi}, \lambda)$ is well defined, since (41) and (42) are well defined. Then by the condition (ii) of Theorem 3.1, and (13), we have

$$
\begin{aligned}
\eta_{1} & =\lambda_{1}-\alpha(x) \\
& =\lambda_{1}-\tilde{\phi}(\bar{\xi}, \lambda) .
\end{aligned}
$$

Although $\eta_{1}=\lambda_{1}-\tilde{\phi}(\bar{\xi}, \lambda)$ cannot be directly measured, $\lambda_{1}-\tilde{\phi}(\theta, \lambda)$ can be directly measured, where $\theta$ is a vector of state variables of the observer (40). Replacing $\lambda_{1}-\tilde{\phi}(\theta, \lambda)$ into $\eta_{1}$ in (32) yields

$$
\begin{aligned}
\dot{\psi} & =W \psi+L\left(\lambda_{1}-\tilde{\phi}(\theta, \lambda)\right) \\
v & =Q \psi .
\end{aligned}
$$

The whole dynamic compensator for $(\mathbf{8})$ consists of (12), (24), (40), and (44), which is written in the following:

$$
\begin{aligned}
\dot{\lambda}_{1} & =\lambda_{2} \\
& \ldots \\
\dot{\lambda}_{l-1} & =\lambda_{l} \\
\dot{\lambda}_{l} & =\sum_{i=1}^{l-1} c_{i} \lambda_{i}+Q \psi \\
\dot{\psi} & =W \psi+L\left(\lambda_{1}-\tilde{\phi}(\theta, \lambda)\right) \\
\dot{\theta} & =\bar{A}_{1} \theta+\bar{L}\left(y-\bar{C}_{1} \theta\right)+\bar{B}_{1} G(0,0) Q \psi \\
u & =\lambda_{1} .
\end{aligned}
$$

It is easy to see that this dynamic compensator is realizable.

Now, we prove that the closed-loop system consisting of (8) and (45) is asymptotically stable.

Let $e=\theta-\bar{\xi}$. Then, from (30) and (40), we have

$$
\begin{aligned}
\dot{e}=\left(\bar{A}_{1}-\bar{L} \bar{C}_{1}\right) e+\bar{B}_{1}[(G(0,0)-G(z, \xi)) Q \psi & \\
& \left.-\bar{\varphi}\left(z, \xi, \eta_{1}, \ldots, \eta_{l}\right)\right] .
\end{aligned}
$$

Since $\lambda_{1}=\eta_{1}+\tilde{\phi}(\bar{\xi}, \lambda)$, we have

$$
\begin{aligned}
\lambda_{1}-\tilde{\phi}(\theta, \lambda) & =\eta_{1}+\tilde{\phi}(\bar{\xi}, \lambda)-\tilde{\phi}(\theta, \lambda) \\
& =\eta_{1}+\tilde{\phi}(\bar{\xi}, \lambda)-\tilde{\phi}(\bar{\xi}+e, \lambda) \\
& =\eta_{1}-D e+R(\bar{\xi}, \lambda, e)
\end{aligned}
$$

where $D=\left.(\partial / \partial \bar{\xi}) \tilde{\phi}(\bar{\xi}, \lambda)\right|_{(\bar{\xi}, \lambda)=0}$, and $R(\bar{\xi}, \lambda, e)=\tilde{\phi}(\bar{\xi}, \lambda)-\tilde{\phi}(\bar{\xi}+$ $e, \lambda)+D e$. It is clear that

$$
R(\bar{\xi}, \lambda, e)=O\left(\|(\bar{\xi}, \lambda, e)\|^{2}\right) \quad R(\bar{\xi}, \lambda, 0)=0 .
$$


Then, by (47), system (44) can be written as

$$
\begin{aligned}
\dot{\psi} & =W \psi+L \eta_{1}-L D e+L R(\bar{\xi}, \lambda, e) \\
v & =Q \psi .
\end{aligned}
$$

It is easy to see that the stability of the closed-loop system (8) and (45) is equivalent to the stability of the system consisting of (29), (46), and (49).

Let $\boldsymbol{w}=\left(\eta^{T}, \psi^{T}, e^{T}\right)^{T}$. Then, the system consisting of (29), (46) and (49) can be written as asymptotically stable. The closed-loop system as the form

$$
\begin{aligned}
\dot{x} & =F\left(x, \eta_{1}\right) \\
\dot{w} & =A w+H(x, w)
\end{aligned}
$$

where $F\left(x, \eta_{1}\right)$ is defined in (34)

$$
A=\left(\begin{array}{ccc}
A_{1} & B_{1} Q & 0 \\
L C_{1} & W & -L D \\
0 & 0 & \bar{A}_{1}-\bar{L} \bar{C}_{1}
\end{array}\right)
$$

and

$$
\begin{aligned}
& H(x, w) \\
& \quad=\left(\begin{array}{c}
B_{1} \varphi\left(z, \xi, \eta_{1}, \ldots, \eta_{l}\right) \\
L R(\bar{\xi}, \lambda, e) \\
\bar{B}_{1}\left[(G(0,0)-G(z, \xi)) Q \psi-B_{1} \bar{\varphi}\left(z, \xi, \eta_{1}, \ldots, \eta_{l}\right)\right]
\end{array}\right) .
\end{aligned}
$$

By using the same method of proving the local asymptotical stability of (37), we can easily prove that system (50) is locally asymptotically stable. This completes the proof of Theorem 3.1.

Remark 3.1: Theorem 3.1 can be extended easily to the case of $m>$ 1 and system (1) has vector relative degree $\left\{r_{1}, r_{2}, \ldots, r_{m}\right\}$.

Remark 3.2: The dimension of a controller for system (1) might be large if we design the controller by following Steps 1-5 exactly. In some circumstances, a controller for (1) with smaller dimension can be designed if we use the approach developed in the above ingeniously. For example, if system (29) can be $N$ th-order approximately stabilized by using a feedback law of the form $v=\beta\left(y, y^{(1)}, \ldots, y^{\left(k_{1}\right)}, \lambda_{1}, \lambda_{2}, \ldots, \lambda_{k_{2}}\right)$, then the dynamic system (32) can be omitted. By this way, the dimension of the stabilizing dynamic controller is reduced.

Before closing this section, we consider a special, but interesting, case of system (8). That is, $g_{0}(z, \xi)=0$. In this case, system (8) becomes

$$
\begin{aligned}
\dot{z} & =f_{0}(z, \xi) \\
\dot{\xi} & =A_{0} \xi+B_{0}\left(F_{0}(z, \xi)+G(z, \xi) u\right) \\
y & =\xi_{1} .
\end{aligned}
$$

For the sake of convenience, we assume that in system (53)

$$
\left.\frac{\partial}{\partial \xi_{i}} f_{0}(z, \xi)\right|_{z=0, \xi=0}=0, \quad i=2,3, \ldots, r
$$

Assumption (54) is not a restriction on system (53), because we can always use a linear coordinate transformation to meet it.

Theorem 3.2: Let (54) hold. If there exist $C^{\infty}$ mappings $\alpha_{1}(z), \alpha_{2}(z), \ldots, \alpha_{r}(z)$ with $\alpha_{i}(0)=0$ for all $1 \leq i \leq r$, defined on some neighborhood of $z=0$, satisfying the following conditions. i) The system

$$
\dot{z}=f_{0}\left(z, \alpha_{1}(z), \alpha_{2}(z), \ldots, \alpha_{r}(z)\right)
$$

is $N$ th-order approximately stable.

ii)

$$
\begin{aligned}
& \alpha_{i+1}(z)=\frac{\partial \alpha_{i}(z)}{\partial z} f_{0}\left(z, \alpha_{1}(z), \alpha_{2}(z), \ldots, \alpha_{r}(z)\right)+\phi_{i}(z) \\
& i=1,2, \ldots, r-1, \text { where } \phi_{i}(z)=O\left(\|z\|^{N+1}\right) .
\end{aligned}
$$

iii) $\alpha_{1}(z)$ is locally uniformly observable with respect to system (53). Then system (53) is locally asymptotically stabilizable by dynamic output feedback.

Proof: Let

$$
\eta_{i}=\xi_{i}-\alpha_{i}, \quad i=1,2, \ldots, r
$$

Then, by using condition ii), system (53) is transformed into the form

$$
\begin{aligned}
\dot{z} & =f_{0}(z, \eta+\alpha(z)) \\
\dot{\eta}_{1} & =\eta_{2}+\beta_{1} \eta_{1}+\gamma_{1}(z, \eta)+\phi_{1}(z) \\
& \ldots \\
\dot{\eta}_{l-1} & =\eta_{l}+\beta_{l-1} \eta_{1}+\gamma_{r-1}(z, \eta)+\phi_{r-1}(z) \\
\dot{\eta}_{l} & =\bar{F}_{0}(z, \eta)+\bar{G}(z, \eta) u \\
y & =\eta_{1}+\alpha_{1}(z)
\end{aligned}
$$

where $\beta_{i}=-\left.\left(\partial \alpha_{i} / \partial z\right)(z) \int_{0}^{1}\left(\partial / \partial \xi_{1}\right) f_{0}(z, \alpha(z)+\sigma \eta) d \sigma\right|_{z=0, \eta=0}$

$$
\begin{aligned}
\gamma_{i}(z, \eta)= & -\left[\frac{\partial \alpha_{i}}{\partial z}(z) \int_{0}^{1} \frac{\partial}{\partial \xi_{1}} f_{0}(z, \alpha(z)+\sigma \eta) d \sigma+\beta_{i}\right] \eta_{1} \\
& -\frac{\partial \alpha_{i}}{\partial z}(z) \sum_{j=2}^{r} \int_{0}^{1} \frac{\partial}{\partial \xi_{j}} f_{0}(z, \alpha(z)+\sigma \eta) d \sigma \eta_{j}, \\
i & =1,2, \ldots, r-1
\end{aligned}
$$

and

$$
\begin{aligned}
\bar{F}_{0}(z, \eta) & =F_{0}(z, \eta+\alpha(z))-\frac{\partial}{\partial z} \alpha_{r}(z) f_{0}(z, \eta+\alpha(z)) \\
\bar{G}(z, \eta) & =G(z, \eta+\alpha(z)) .
\end{aligned}
$$

Due to (54) and (58), we have

$$
\gamma_{i}(z, 0)=0, \gamma_{i}(z, \eta)=O\left(\|(z, \eta)\|^{2}\right), i=1,2, \ldots, r-1 .
$$

By using the technique developed in the proof of Theorem 3.1, we can construct a dynamic compensator for system (57) such that the closed-loop system is asymptotically stable. Since the construction is very similar to that in Theorem 3.1, details of the construction are omitted. The proof is completed.

Remark 3.3: The dimension of a controller constructed by using Theorem 3.2, in general, may be less than that of Theorem 3.1 (see Example 4.1).

Remark 3.4: Theorem 3.2 provides a novel solution to output feedback stabilization of systems with strong nonlinearities. Nevertheless, it should be noted that Assumption (ii) of Theorem 3.2, though crucial, may be hard to check.

\section{AN Illustrative EXAMPLE}

We illustrate our results by means of an elementary example. 
1) Example 4.1: Consider the system

$$
\begin{aligned}
& \dot{z}=z^{4}+z \xi \\
& \dot{\xi}=z^{2}+u \\
& y=\xi
\end{aligned}
$$

where $z \in R, \xi \in R ; y$ is the output and $u$ is the input.

First, it is shown that system (61) is not stabilizable by static output feedback. Indeed, if (61) is stabilizable by static output feedback, then there exists a $C^{\infty}$ feedback $u=\mu(\xi)$ with $\mu(0)=0$ such that the closed-loop system

$$
\begin{aligned}
& \dot{z}=z^{4}+z \xi \\
& \dot{\xi}=z^{2}+\mu(\xi) \\
& y=\xi
\end{aligned}
$$

is asymptotically stable. We can assume without loss of generality that $\mu(\xi)$ is defined on $(-\infty, \infty)$. Consider the region $D=\{(z, \xi) \mid z>$ $0, \xi>0\}$. Let $(z(t), \xi(t))^{T}$ be a solution of (62). It is easy to see that $D$ is an invariant region of (62) and $\dot{z}(t)>0$ if $(z(t), \xi(t))^{T} \in D$. This implies that system (62) is not stable. This contradiction shows that system (61) is not stabilizable by static output feedback.

Then notice that system (61) has relative degree 1, and that the system is nonminimum-phase. Indeed, the zero dynamics of system (61) is

$$
\dot{z}=z^{4}
$$

which clearly is not stable. It is also of interest to note that the state variables of system (61) are not locally completely uniformly observable (see, for instance, [13]). Therefore, system (61) cannot be stabilized by existing methods (see, for instance, [3]-[5], [13], and [15]). Now, we use the approach proposed in this note to derive a dynamic output-feedback controller that stabilizes (61).

In this example, $f_{0}(z, \xi)=z^{4}+z \xi$. Let $\alpha_{1}(z)=-z^{2}$. It is easy to see that the system

$$
\dot{z}=f_{0}\left(z, \alpha_{1}(z)\right)=-z^{3}+z^{4}
$$

is third-order approximately stable. On the other hand, $\alpha_{1}(z)$ can be expressed as

$$
\alpha_{1}(z)=-\dot{y}+u
$$

that is, $\alpha_{1}(z)$ is locally uniformly observable. Therefore, system (61) satisfies the conditions of Theorem 3.2. Then we can use the design technique developed in Section III to construct a dynamic compensator for (61) as follows

$$
\begin{aligned}
\dot{\lambda}_{1} & =-y+\lambda_{1}-2 \theta_{2} \\
\dot{\theta}_{1} & =\theta_{2}+\left(y-\theta_{1}\right) \\
\dot{\theta}_{2} & =-y+\lambda_{1}-2 \theta_{2}+\left(y-\theta_{1}\right) \\
u & =\lambda_{1} .
\end{aligned}
$$

\section{CONCLUSION}

Based on the concepts of approximate stability and local uniform observability of feedback control law, a novel constructive approach for dynamic output feedback stabilization of nonlinear systems is proposed. The systems considered are of a wide class, which may have unstable zero dynamics and may be linearly uncontrollable and/or linearly unobservable. To extend the proposed approach to the problem of semiglobal stabilization or global stabilization is an interesting topic for further study.

\section{REFERENCES}

[1] D. Aeyels, "Stabilization of a class of nonlinear systems by a smooth feedback," Syst. Control Lett., vol. 5, pp. 289-294, 1985.

[2] S. Behtash and S. S. Sastry, "Stabilization of nonlinear systems with uncontrollable linearization," IEEE Trans. Autom. Control, vol. 33, no. 6, pp. 585-590, Jun. 1988.

[3] C. I. Byrnes and A. Isidori, "New results and examples in nonlinear feedback stabilization," Syst. Control Lett., vol. 12, pp. 437-442, 1989.

[4] P. Chen, H. Qin, D. Cheng, and Y. Hong, "Stabilization of minimum phase nonlinear systems by dynamic output feedback," IEEE Trans. Autom. Control, vol. 45, no. 12, pp. 2331-2335, Dec. 2000.

[5] P. Chen, H. Qin, and J. Huang, "Local stabilization of a class of nonlinear systems by dynamic output feedback," Automatica, vol. 37, pp. 969-981, 2001.

[6] D. Cheng and C. Martin, "Stabilization of nonlinear systems via designed center manifold," IEEE Trans. Autom. Control, vol. 46, no. 9, pp. 1372-1383, Sep. 2001.

[7] F. Esfandiari and H. K. Khalil, "Output feedback stabilization of full linearizable systems," Int. J. Control, vol. 56, pp. 1007-1037, 1992.

[8] W. Hahn, Stability of Motion. New York: Springer-Verlag, 1967.

[9] A. Isidori, Nonlinear Control Systems, 3rd ed. London, U.K.: Springer-Verlag, 1995.

[10] — - "A tool for semiglobal stabilization of uncertain non-minimumphase nonolinear systems via output feedback," IEEE Trans. Autom. Control, vol. 45, no. 10, pp. 1817-1827, Oct. 2000.

[11] L. Praly and Z. P. Jiang, "Stabilization by output feedback for systems with ISS inverse dynamics," Syst. Control Lett., vol. 21, pp. 19-33, 1993.

[12] C. Qian and W. Lin, "Practical output tracking of nonlinear systems with uncontrollable unstable linearization," IEEE Trans. Autom. Control, vol. 47, no. 1, pp. 21-36, Jan. 2002.

[13] A. Teel and L. Praly, "Global stabilizability and observability imply semi-global stabilizability by output feedback," Syst. Control Lett., vol. 22, pp. 313-325, 1994.

[14] _ _ "Tools for semi-global stabilization by partial state and output feedback," SIAM J Control Optim., vol. 33, pp. 1443-1488, 1995.

[15] A. Tornambè, "Output feedback stabilization of a class of non-minimum phase nonlinear systems," Syst. Control Lett., vol. 19, pp. 193-204, 1992.

[16] J. Tsinias, "Partial-state global stabilization for general triangular systems," Syst. Control Lett., vol. 24, no. 2, pp. 139-145, 1995.

The details are omitted for want of space. 